\title{
Toward dynamical systems medicine: personalized and preventive strategies
}

\author{
"By focusing on the disease 'roots', the essential goal of personalized \\ and systems medicine is to fine-tune the imbalance in the whole \\ mind-body system."
}

First draft submitted: 19 April 2017; Accepted for publication: 23 May 2017; Published online: 1 September 2017

Keywords: complex adaptive systems $\bullet$ dynamical medicine $\bullet$ inflammation $\bullet$ mind-body - networks $\bullet$ personalized medicine $\bullet$ prevention $\bullet$ self-regulation $\bullet$ spatiotemporal $\bullet$ systems medicine

\section{Systems medicine: the dynamical features of the complex adaptive systems}

Facing the tremendous challenges in healthcare such as the skyrocketing costs and unsatisfied patient outcomes, systems medicine may provide the hope by emphasizing on holistic, personalized and preventive strategies. The development of systems medicine may revolutionize the reductionismbased and disease-centered agenda toward a dynamical human-centered paradigm [1].

This schema would embrace the concepts of complex adaptive systems (CASs) for interpreting the 'whole mind-body system' toward a novel and comprehensive understanding of health and diseases [1]. An important concept is the 'emergence' of behaviors or symptoms as the results from the overall processes and dynamical interconnections. For example, inflammation has been deemed as the critical pathogenesis in various diseases including depression, diabetes, cardiovascular diseases and cancer [2]. Systemic inflammation may result from the multidirectional interactions in the microbiota-gut-brain axis, but cannot be explained with a single cytokine [3].

Another concept is 'adaptation', which is critical in responses to social and environmental stressors for maintaining psychophysiological coherence [1]. Pathologically, it can be used to describe the dynamical processes from disease onset to progression.
The coevolutionary activities may also lead to multiple drug resistance in the treatments of infectious diseases and cancers [4]. Such issues can be tackled using different therapeutic protocols under different conditions to avoid the adaptation.

In addition, 'self-organization' and 'robustness' are the essential features of living CASs for maintaining homeostasis and disease healing [1]. The applications of such concepts can concentrate on promoting the self-adjustment of abnormal functions, rather than simply 'winning' the wars on diseases by killing viruses or cancer cells that often results in adverse reactions.

Under such guidelines, the therapeutic targets would transform from pathogenic molecules to host-pathogen interactions, from cancer cells to inflammatory networks at various system levels and time points. For instance, oxidation and inflammation are intertwined feedback loops in various disorders including anxiety and premature aging [5]. The focus of the relevant treatments would be promoting the abilities of self-regulation toward normal neuroimmune functions to relieve the oxidative-inflammatory conditions.

Furthermore, the self-organization and robust activities may affect the interactions among different system levels [1]. The hierarchically organized networks are composed of interconnected genes, cells, tissues, organs, individuals and populations [6].

\section{Qing Yan}

PharmTao, Santa Clara, CA 95056-5672, USA

Tel.: +1 4083909479

qyan@pharmtao.com
Future $\%$
Medicine ${ }_{\text {part of }}$ fsg 
The process at one level can influence or manifest as the behaviors or symptoms at the other levels. For example, the sign of sunburn is an alteration at the organism level with DNA damages in skin tissues that can be detected at molecular and cellular levels. The systems-based approaches should cover the across-level inter-relationships including the genes-cells-human-environment networks [3].

Such interconnections also address the concept of 'nonlinearity', as many interactions do not have linear results. For instance, the continuous increase of drug dosages may lead to serious adverse effects but not better outcomes [1]. Embracing these concepts of CASs in personalized medicine would facilitate clinical practice "to bring the right interventions to the right people with the right dosages and intensities at the right time" [1].

"The emphasis of the term 'dynamical' in medicine may underscore the features of adaptation and robustness in the living complex adaptive systems."

\section{Personalized medicine: focusing on the roots toward the dynamical balance}

The development of personalized and systems medicine requires the identification of the 'roots' of diseases, in other words, the alterations in the whole mind-body system, rather than just diseases themselves. Different diseases including Type 2 diabetes and cardiovascular diseases often share the same causes including unhealthy dietary habits and lifestyles. Chronic inflammation has been considered the common mechanism underlying various disorders including obesity, kidney disorders, Alzheimer's disease, heart diseases and cancer [2]. The same therapy such as IL1 $\beta$ neutralization has been found useful in apparently unconnected diseases including gout, heart failure and cancer [7]. In addition, different combinations of drugs may lead to the same results, while the same therapeutic protocols often have different outcomes in different patients.

These observations support the strategies of 'treating the same disease differently' and 'treating different diseases in similar ways'. Such strategies have several implications for personalized medicine. First, the same disease in different patients should be treated differently if it is caused by different mechanisms. Individual characteristics should also be considered, including age, gender, race, body sizes and temperaments. Second, the same disease in the same patient during different stages should be treated differently. Third, even though the diseases and symptoms are different in the same or different patients, they may share the similar mechanisms such as the same inflammatory pathways and can be treated using similar protocols.
Such strategies may represent the substantial transformation from disease-centered medicine to humancentered care. For instance, the human microbiota is an ecosystem involved in the neuroimmune functions, drug and carcinogen neutralization, intestinal motility and visceral perception [8]. With the understanding of its dynamical features, the microbiota-gut-brain axis can become the key target for treating a broad range of inflammation-associated diseases, for example, using probiotics or prebiotics [9].

By focusing on the disease 'roots', the essential goal of personalized and systems medicine is to fine-tune the imbalance in the whole mind-body system. The promotion of the normal functions of the overall selfregulatory networks [10] at various spatial levels and temporal scales would help restore homeostasis and the dynamical 'balance of health'.

For example, molecular and cellular mechanisms of the imbalance between vascular damage and repair have been identified in aging and age-related diseases including diabetes, atherosclerosis, neurodegenerative disorders and cancer [11]. Early identifications of the biomarkers indicating such imbalance and the corresponding therapeutics have been suggested as the more effective methods for the comorbidities than simply 'treating actually vascular diseases' [11].

With the failure of the reductionism-based 'onedrug-fits-all' and 'one gene-one disease-one drug' schemes, the treatment of the disease 'roots' would become more meaningful for personalized medicine. Recent developments in this direction include drug repositioning that expands the utilizations of existent drugs to the conditions beyond the initial therapeutic boundaries [3]. Furthermore, the strategy of drug combinations may target the mutations or escape pathways from various points to avoid drug resistance in complex disorders including cancer [12].

Drug repositioning has opened a cost-effective path with higher efficiency by saving the expenditures and time from drug discovery and clinical trials [3]. Translational bioinformatics strategies including data integration may facilitate the transcriptomic profiling and 'connectivity mapping' of the gene/pathway-drug networks $[3,13]$. Systematic network-based computational methods would enable the modeling of the interactions by mining the large-scale data from electronic health records and disease/drug expression profiles [14].

\section{Dynamical medicine: the integrative strategies for prediction, prevention and treatment}

In conclusion, the accomplishment of personalized medicine relies on the comprehension of the 'dynamical' features, especially the across-scale spatiotemporal 
profiling from molecules to organisms, from nanoseconds to seasons [1]. The highlight of the word 'systems' in medicine may address the 'roots' of health and diseases including the attributes of emergence, nonlinearity and self-organization. The emphasis of the term 'dynamical' in medicine may underscore the features of adaptation and robustness in the living CASs.

As an essential property of life from mitochondria to memory, the dynamical patterns request shifting and systemic targets for disease interventions. Based on such perception, prediction and prevention in personalized medicine have several layers of meanings.

The first layer is to prevent the onset of diseases, especially among those populations with high risks and susceptibilities. The second layer is to predict and prevent the disease progression to the next stages or other body areas. The third layer is effective follow-ups to prevent the potential recurrence.

To achieve these goals, dynamical systems-based profiles can be established to incorporate the temporal and evolving components including circadian rhythms, nonlinear time-series patterns and feedback networks [3]. For example, the 'dynamical network biomarkers' can illustrate the cell as a CASs with the varying molecular networks to represent the early warning signals of disease initiation and progression [15]. This strategy can be expanded to include across-scale spatiotemporal factors to replace the static portraits in the conventional diagnosis.

Among these factors, the rhythmic variability such as heart rate dynamics can be especially important for the complex networks. Such mechanisms may connect the omics at various levels from mitochondrial

\section{References}

1 Yan Q. From pharmacogenomics and systems biology to personalized care: a framework of systems and dynamical medicine. Methods Mol. Biol. 1175, 3-17 (2014).

2 Yan Q. Psychoneuroimmunology: Systems Biology Approaches to Mind-body Medicine. Springer, NY, USA (2016).

3 Yan Q. Translational Bioinformatics and Systems Biology Methods for Personalized Medicine. Academic Press, London, UK (2017).

4 Avner BS, Fialho AM, Chakrabarty AM. Overcoming drug resistance in multi-drug resistant cancers and microorganisms: a conceptual framework. Bioengineered 3, 262-270 (2012).

5 Vida C, González EM, De la Fuente M. Increase of oxidation and inflammation in nervous and immune systems with aging and anxiety. Curr. Pharm. Des. 20, 4656-4678 (2014).

6 Govindaraju DR, Annaswamy AM. Application of smart infrastructure systems approach to precision medicine. Appl. Transl. Genom. 7, 40-44 (2015). bioenergetics to neuroimmune functions [16]. For example, aging and associated disorders have been related to the loss of the rhythmic variability showing at multiple scales with altered functions at the smaller scale and detectable diagnostic traits at the larger scale [16]. The profiling of dynamical systems-based biomarkers may not only track the trajectory of health and diseases, but also enable presymptomatic diagnosis and prognosis for precise prediction and prevention.

Integrative interventions based on such biomarkers or profiles can be designed for the practice of dynamical systems medicine, including mind-body approaches, lifestyle changes and personalized nutrition [17]. For example, food components may interact with epigenetic factors with powerful protective and preventive potentials against inflammation in aging and age-related diseases [18]. Moreover, circadian rhythms have profound effects on xenobiotic metabolism and detoxification processes [19]. However, even with strong evidences, chronotherapy including the 'time-of-day' administration still needs more attention for personalized therapeutic regimens to reduce adverse effects and promote the efficacy [3,19].

\section{Financial \& competing interests disclosure}

The author has no relevant affiliations or financial involvement with any organization or entity with a financial interest in or financial conflict with the subject matter or materials discussed in the manuscript. This includes employment, consultancies, honoraria, stock ownership or options, expert testimony, grants or patents received or pending, or royalties.

No writing assistance was utilized in the production of this manuscript.

7 Dinarello CA. Blocking interleukin- $1 \beta$ in acute and chronic autoinflammatory diseases. J. Intern. Med. 269, 16-28 (2011).

8 Elisei C, de Castro AP. Insight into role of microbiota-gutbrain peptides as a target for biotechnology innovations. Front. Biosci. (Elite Ed.) 9, 76-88 (2017).

9 Kanauchi O, Andoh A, Mitsuyama K. Effects of the modulation of microbiota on the gastrointestinal immune system and bowel function. J. Agric. Food Chem. 61, 9977-9983 (2013).

10 Goldman AW, Burmeister Y, Cesnulevicius K et al.Bioregulatory systems medicine: an innovative approach to integrating the science of molecular networks, inflammation, and systems biology with the patient's autoregulatory capacity? Front. Physiol. 6, 225 (2015).

11 Madonna R, Novo G, Balistreri CR. Cellular and molecular basis of the imbalance between vascular damage and repair in ageing and age-related diseases: as biomarkers and targets for new treatments. Mech. Ageing Dev. 159, 22-30 (2016). 
12 Ryall KA, Tan AC. Systems biology approaches for advancing the discovery of effective drug combinations. J. Cheminform 7, 7 (2015).

13 Powell TR, Murphy T, Lee SH et al. Transcriptomic profiling of human hippocampal progenitor cells treated with antidepressants and its application in drug repositioning. J. Psychopharmacol. (Oxford) 31, 338-345 (2017).

14 Jadamba E, Shin MA. Systematic framework for drug repositioning from integrated omics and drug phenotype profiles using pathway-drug network. Biomed. Res. Int. 2016, 7147039 (2016).

15 Vafaee F. Using multi-objective optimization to identify dynamical network biomarkers as early-warning signals of complex diseases. Sci. Rep. 6, 22023 (2016).
16 Sturmberg JP, Bennett JM, Picard M, Seely AJE. The trajectory of life. Decreasing physiological network complexity through changing fractal patterns. Front. Physiol. 6, 169 (2015).

17 Yan Q. The role of psychoneuroimmunology in personalized and systems medicine. Methods Mol. Biol. 934, 3-19 (2012).

18 Szarc vel Szic K, Declerck K, Vidaković M, Vanden Berghe $\mathrm{W}$. From inflammaging to healthy aging by dietary lifestyle choices: is epigenetics the key to personalized nutrition? Clin. Epigenetics 7, 33 (2015).

19 Selfridge JM, Gotoh T, Schiffhauer S et al. Chronotherapy: intuitive, sound, founded... but not broadly applied. Drugs 76, 1507-1521 (2016). 